\title{
THE QUANTUM MECHANICAL SPHERICAL PENDULUM
}

\author{
R. CUSHMAN AND J. J. DUISTERMAAT
}

\begin{abstract}
In this announcement we describe the asymptotic behavior of the spectrum of the quantum mechanical spherical pendulum as Planck's constant tends to zero.
\end{abstract}

We begin by discussing

1. The classical spherical pendulum $[6,4]$. As a Hamiltonian system the spherical pendulum has a configuration space

$$
S^{2}=\left\{q=\left(q_{1}, q_{2}, q_{3}\right) \in \mathbf{R}^{3} \mid 1=q_{1}^{2}+q_{2}^{2}+q_{3}^{2}=\langle q, q\rangle\right\}
$$

and a phase space

$$
T^{*} S^{2}=\left\{(q, p) \in \mathbf{R}^{3} \times \mathbf{R}^{3} \mid\langle q, q\rangle=1,\langle q, p\rangle=0\right\} .
$$

The standard symplectic form $\sum_{i=1}^{3} d q_{i} \wedge d p_{i}$ on $\mathbf{R}^{3} \times \mathbf{R}^{3}$ when restricted to $T^{*} S^{2}$ gives the canonical symplectic form on $T^{*} S^{2}$. The dynamics is given by the Hamiltonian function

$$
E: T^{*} S^{2} \rightarrow \mathbf{R}:(q, p) \rightarrow \frac{1}{2}\langle p, p\rangle+q_{3} .
$$

Since $E$ is invariant under rotations about the $q_{3}$ axis lifted to $T^{*} S^{2}$, the function

$$
L: T^{*} S^{2} \rightarrow \mathbf{R}:(q, p) \rightarrow q_{1} p_{2}-q_{2} p_{1}
$$

is an integral of the Hamiltonian vectorfield $X_{E} . L$ is the $q_{3}$-component of angular momentum. Thus the flows $\phi_{t}^{E}$ and $\phi_{t}^{L}$ of $X_{E}$ and $X_{L}$, respectively, commute. Hence the spherical pendulum is completely integrable.

Consider the energy momentum mapping

$$
\mathscr{E} \mathscr{M}: T^{*} S^{2} \rightarrow \mathbf{R}^{2}:(q, p) \rightarrow(E(q, p), L(q, p))
$$

(see Figure 1). Suppose that $(e, l) \in \mathscr{R}$, the set of regular values of $\mathscr{C} \mathscr{M}$, which is the shaded region in Figure 1 excluding $(1,0)$ and boundary curves. Then $\mathscr{E} \mathscr{M}^{-1}(e, l)=E^{-1}(e) \cap L^{-1}(l)$ is a compact, connected, smooth, twodimensional submanifold of $T^{*} S^{2}$. On $\mathscr{E} \mathscr{M}^{-1}(e, l)$ we have an $\mathbf{R}^{2}$ action defined by

$$
\Phi: \mathbf{R}^{2} \times \mathscr{E} \mathscr{M}^{-1}(e, l) \rightarrow \mathscr{E} \mathscr{M}^{-1}(e, l):\left(\left(t_{1}, t_{2}\right), m\right) \rightarrow \phi_{t_{1}}^{E} \circ \phi_{t_{2}}^{L}(m)
$$

which is transitive. Therefore the isotropy group

$$
P(e, l)=\left\{\left(T_{1}, T_{2}\right) \in \mathbf{R}^{2} \mid \phi_{T_{1}}^{E} \circ \phi_{T_{2}}^{L}=\mathrm{id}_{\mathscr{E}, \mathscr{K}^{-1}(e, l)}\right\}
$$

Received by the editors January 14, 1988.

1980 Mathematics Subject Classification (1985 Revision). Primary 58G25, 81C05. 


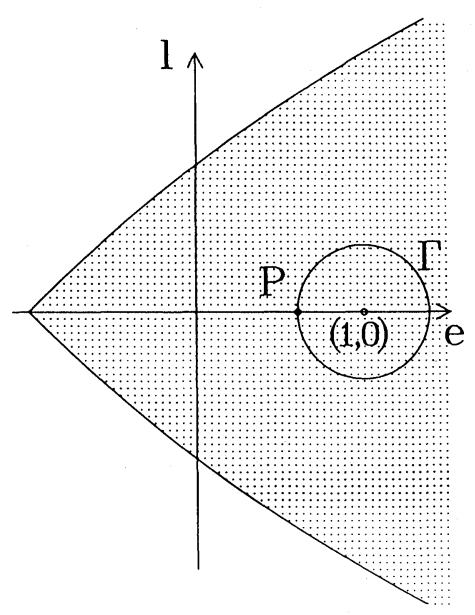

FIGURE 1

is a lattice in $\mathbf{R}^{2}$ called the period lattice. Consequently $\mathscr{C} \mathscr{M}^{-1}(e, l)$ is a two-dimensional torus $\mathbf{R}^{2} / P(e, l)$.

We now discuss the construction of action angle co-ordinates. The basic idea is to find smooth functions $a_{j}=a_{j}(e, l), j=1,2$, so that the flow of $X_{a_{j}} \mid \mathscr{E} \mathscr{M}^{-1}(e, l)$ is periodic of period $2 \pi$. Let $\mathscr{T}^{(j)}(e, l)=\left(\tau_{1}^{(j)}, \tau_{2}^{(j)}\right), j=1,2$, be a basis of $P(e, l)$ which depends smoothly on $(e, l)$. Then

$$
t \rightarrow \Phi^{t \mathscr{T}^{(j)} / 2 \pi}\left|\mathscr{E} \mathscr{M}^{-1}(e, l)=\phi_{t \tau_{1}^{(j)} / 2 \pi}^{E} \circ \phi_{t \tau_{2}^{(j)} / 2 \pi}^{L}\right| \mathscr{E} \mathscr{M}^{-1}(e, l)
$$

is a $2 \pi$ periodic flow of the vectorfield

$$
X_{a_{j}}=\frac{\partial a_{j}}{\partial e} X_{E}+\frac{\partial a_{j}}{\partial l} X_{L}
$$

on $\mathscr{E} \mathscr{M}^{-1}(e, l)$, provided that

$$
\frac{\partial a_{j}}{\partial e}=\frac{\tau_{1}^{(j)}}{2 \pi} \quad \text { and } \quad \frac{\partial a_{j}}{\partial l}=\frac{\tau_{2}^{(j)}}{2 \pi} .
$$

Hence, choosing a smooth basis of the period lattice determines $d a_{j}, j=1,2$, uniquely. Since $X_{L} \mid \mathscr{E} \mathscr{M}^{-1}(e, l)$ has a $2 \pi$ periodic flow, we may choose $a_{1}=l$. To find the second action function $a_{2}$ we need some facts about the motion of the spherical pendulum (see Figure 2).

(1) The image of an integral curve $\gamma_{1}$ of $X_{E}$ under the bundle projection $\pi: T^{*} S^{2} \rightarrow S^{2}:(q, p) \rightarrow q$ is bounded between two circles of constant latitude $q_{3}^{ \pm}=q_{3}^{ \pm}(e, l)$.

(2) The $q_{3}$-component of $\pi\left(\gamma_{1}\right)$ is periodic of period $T=T(e, l)$.

(3) $\pi^{-1}(\mathscr{C})=\gamma_{2}$ is a cross section for the flow of $X_{E} \mid \mathscr{E} \mathscr{M}^{-1}(e, l)$ which is an integral curve of $X_{L}$.

Therefore if we start at $Q_{1}=\pi\left(m_{1}\right)$ on $\mathscr{C}, \gamma_{1}$ returns to $\mathscr{C}$ after time $T$. During this time $Q_{1}$ has moved along $\mathscr{C}$ through an angle $\Delta \theta(e, l)$ to 


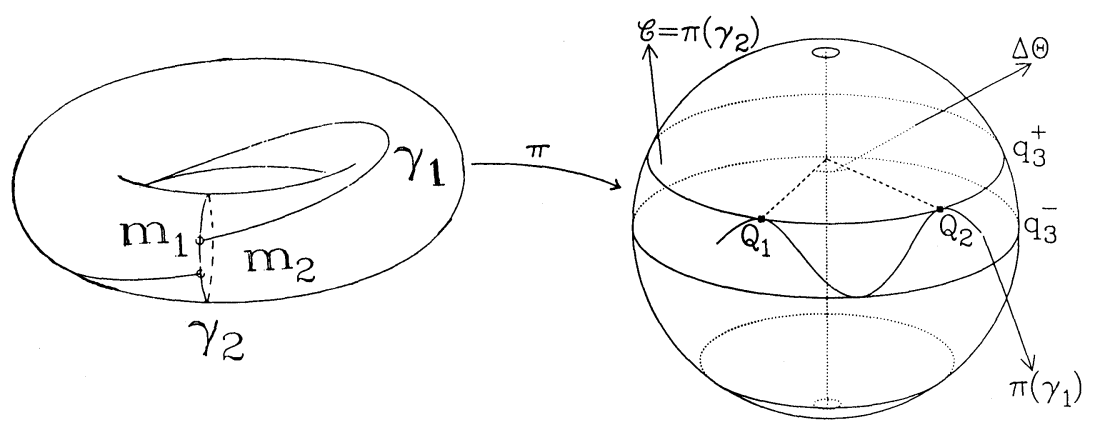

FIGURE 2

$Q_{2}=\pi\left(m_{2}\right)$. Consequently for any $m$ on $\gamma_{2}$ we have $\phi_{T / 2 \pi}^{E}(m)=\phi_{\Delta \theta / 2 \pi}^{L}(m)$. In other words we may choose

$$
a_{2}=\frac{T}{2 \pi} E-\frac{\Delta \theta}{2 \pi} L .
$$

For then $X_{a_{2}}$ has a $2 \pi$-periodic flow on $\mathscr{C}^{-1}(e, l)$. Since

$$
\phi_{T / 2 \pi}^{E}(m)=\phi_{\Delta \theta+n 2 \pi / 2 \pi}^{L}(m)
$$

for all $n \epsilon \mathbf{Z}$ we may make another choice for the second action function, namely:

$$
\tilde{a}_{2}=\frac{T}{2 \pi} E-\left(\frac{\Delta \theta}{2 \pi}+n\right) L .
$$

There are no other possibilities. A classical calculation shows that

$$
a_{2}(e, l)=2 \int_{q_{3}^{-}}^{q_{3}^{+}}\left[2(e-z)\left(1-z^{2}\right)-l^{2}\right] d z,
$$

where $2\left(e-q_{3}^{ \pm}\right)\left(1-q_{3}^{ \pm 2}\right)-l^{2}=0$ and $q_{3}^{ \pm} \in[-1,1]$.

Now we are in position to describe the monodromy of the spherical pendulum. Look at Figure 1. Let $\Gamma$ be a circle centered at $(1,0)$ contained in $\mathscr{R}$, the set of regular values of $\mathscr{E} \mathscr{M}$. Let $p=\left(e_{0}, 0\right) \in \Gamma \cap\{l=0\}$ with $e_{0}<1$. Choose a period lattice $P\left(e_{0}, 0\right)$ so that $n=0$ in (1). Next select a family of period lattices $P(e, l)$ which depend smoothly on $(e, l)$ as $(e, l)$ traverses $\Gamma$ clockwise. When $(e, l)$ returns to $\left(e_{0}, 0\right)$ after one circuit of $\Gamma$, we find that $n=1$ in (1). Thus $a_{2}$ is a multivalued real analytic function of $(e, l) \in \mathscr{R}$. This implies that one cannot find action angle variables in a neighborhood of $(1,0)$.

2. The quantum mechanical spherical pendulum. The Schrödinger operator which quantizes $E=\frac{1}{2}\langle p, p\rangle+q_{3}$ is $\mathscr{E}=-h^{2} \Delta / 2+\mathscr{V}$, where $h$ is Planck's constant, $\Delta$ is the Laplace operator on $S^{2}$, and $\mathscr{V}$ is the operator of multiplication by $q_{3}$. $\mathscr{E}$ commutes with the operator $\mathscr{L}$ corresponding to $L$. $\mathscr{E}$ and $\mathscr{L}$ are selfadjoint. $\mathscr{E}$ has discrete spectrum with finite multiplicity, since it is elliptic. Because $\mathscr{L}$ commutes with $\mathscr{E}, \mathscr{L}$ leaves the eigenspaces of $\mathscr{E}$ invariant. Thus the eigenfunctions of $\mathscr{L}$ in the eigenspaces of $\mathscr{E}$ are 
common eigenfunctions for $\mathscr{L}$ and $\mathscr{E}$, that is, $\mathscr{E}\left(\Psi_{k}\right)=E_{k} \Psi_{k}$ and $\mathscr{L}\left(\Psi_{k}\right)=$ $L_{k} \Psi_{k}$. The question we address is: what does the spectrum $\left(E_{k}, L_{k}\right)$ look like as $h \rightarrow 0$ ?

Using oscillatory integrals [5, pp. 228-232] supported on the Lagrangian tori $\mathscr{E} \mathscr{M}^{-1}(e, l)$, which is given by the intersection of the level sets $E^{-1}(e)$ and $L^{-1}(l)$ of the principal symbol $E$ of $\mathscr{E}$ and $L$ of $\mathscr{L}$, respectively, one finds functions $\psi_{m, n}, m \in \mathbf{Z}_{\geq}, n \in \mathbf{Z}$, such that

$$
\mathscr{E}\left(\psi_{m, n}\right)=E_{m, n} \psi_{m, n}+O\left(h^{2}\right) \quad \text { and } \quad \mathscr{L}\left(\psi_{m, n}\right)=h n \psi_{m, n}
$$

where the Bohr-Sommerfeld rule

$$
a_{2}\left(E_{m, n}, h n\right)=h\left(m+\frac{1}{2}\right)
$$

holds and $\left(E_{m, n}, h n\right)$ is close to a regular value of $\mathscr{E} \mathscr{M}$. From this asymptotic construction we obtain candidate eigenvalues, called the quasiclassical spectrum, at the "lattice" $(e, l)$ where the action variables are quantized by

$$
\begin{aligned}
& a_{1}=a_{1}(e, l)=h n, \quad n \in \mathbf{Z}, \\
& a_{2}=a_{2}(e, l)=h\left(m+\frac{1}{2}\right), \quad m \in \mathbf{Z}_{\geq} .
\end{aligned}
$$

A straightforward computation (done by Professor M. van Veldhuizen of the Free University of Amsterdam) gives Figure 3 which displays the quasiclassical spectrum.

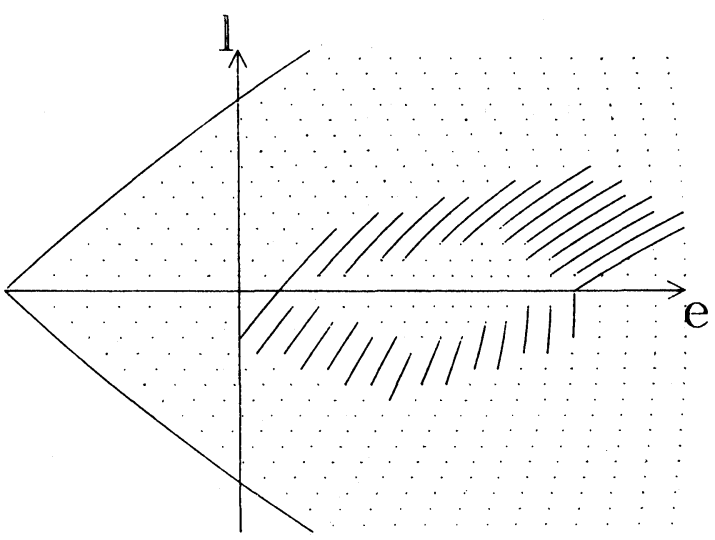

FIGURE 3

This spectrum has monodromy. More precisely, every real analytic function of $(e, l) \in \mathscr{R}$ which is (1) not a function of $l$ alone and (2) has level curves passing through the quasiclassical spectrum, is not single valued. The multivaluedness is the same as that of $a_{2}$ in the classical spherical pendulum. To see the quantum mechanical monodromy we draw a local (dark) real analytic curve through the dots on three or more adjacent rows. Then we propagate this level curve by drawing parallel (light) curves through neighboring dots on 
two adjacent rows. This is possible since the eigenvalues of $\mathscr{E}$ and $\mathscr{L}$ depend real analytically on $(e, l)$. Repeating this process one sees that the level curves do not match up globally. In fact four dots : : lying on adjacent rows and neighboring level lines become $\ldots$ This change in the local lattice structure of the quasiclassical spectrum is the quantum mechanical monodromy of the spherical pendulum.

The asymptotic construction implies that at distance $O\left(h^{2}\right)$ from the points $\left(E_{m, n} n h\right)$ in $\mathscr{R}$, there are eigenvalues of $\mathscr{E}$ and $\mathscr{L}$. But there could be many others which are not given by the asymptotic construction. This being so the $\psi_{m, n}$ would not necessarily be close to an eigenfunction of $\mathscr{E}$ and $\mathscr{L}$. If $\mathscr{E}$ and $\mathscr{L}$ had eigenvalues which were very close to one another then $\psi_{m, n}$ could be close to an arbitrary linear combination of eigenfunctions of $\mathscr{E}$ and $\mathscr{L}$. For this reason $\mathrm{Arnol}^{\prime} \mathrm{d}$ [1] calls such eigenfunctions quasimodes. In the case of the spherical pendulum, there are no quasimodes, that is, there are no other eigenvalues nearby the ones given by the asymptotic construction. Hence $\psi_{m, n}$ are at distance $O(h)$ from exact eigenfunctions. One of us (J.J.D.) has checked this by studying the ordinary differential operator

$$
-\frac{h^{2}}{2} \frac{d}{d z}\left(1-z^{2}\right) \frac{d}{d z}+\frac{l^{2}}{2\left(1-z^{2}\right)}+z \quad \text { for } l=n h, z=q_{3} \in(-1,1),
$$

which one obtains if $\mathscr{E}$ is restricted to an eigenspace of $\mathscr{L}$. The boundary condition that $e^{i n \theta} \psi_{m, n}(\theta, z)$ is in $L^{2}\left(S^{2}\right)$ becomes the Dirichlet conditions $z(-1)=z(1)=0$ for (6). It turns out that the classical Sturm-Liouville theory goes through for (6) and gives the desired asymptotics as $h \rightarrow 0$. (See $[2,3]$ for another proof of the nonexistence of quasimodes.)

Acknowledgments. One of us (R.C.) would like to thank Professor V. Guillemin of MIT and Professor J. Sniatycki of the University of Calgary for stimulating conversations on this topic. Professor Guillemin also had the idea that quantum mechanical monodromy was due to the change in the local lattice structure of the quasiclassical spectrum. We would also like to thank Professor M. van Veldhuizen of the Free University of Amsterdam for doing the computer graphics for the figures in this announcement.

\section{REFERENCES}

1. V. I. Arnol'd, Modes and quasimodes, J. Funct. Anal. Appl. 6 (1972), 94-101.

2. A. M. Charbonnel, Comportement semi-classique du spectre conjoint d'opérateurs pseudo differentiels qui commutent, Seminar Equations aux Derivees Partielles, Univ. de Nautes, Dept. de Math., 1987.

3. __ preprint, Asymptotic Analysis (to appear).

4. R. Cushman, Geometry of the energy momentum mapping of the spherical pendulum, Centrum voor Wiskunde en Informatica Newsletter 1 (1983), 4-18.

5. J. J. Duistermaat, Oscillatory integrals, Lagrange immersions and unfolding of singularities, Comm. Pure Appl. Math. 27 (1974), 207-281.

6. __ On global action-angle variables, Comm. Pure Appl. Math. 33 (1980), 687-706.

\section{MATHEMATICS Institute, RiJKSUNIVERsiteit UTRECht, UTRECHT, The} NETHERLANDS 
